\title{
Multiculturalism in Healthcare: A Review of Current Research into Diversity Found in the Healthcare Professional Population and the Patient Population
}

Nicole Tan,' Shuangyu Li.'

\begin{abstract}
Culture plays a key role in the way health is perceived and the way healthcare is used. It has been seen that there are many barriers to accessing healthcare, particularly for those who come to the UK with a different background language, religion and healthcare structure. A literature review and content analysis has been performed using 60 articles, selected from the databases PubMed, Scopus and Web of Science, in order to expose how diverse the patient and healthcare professional population truly are, the number of barriers to healthcare and how one can improve access to become culturally competent. Although this is only a small sample of the material present, it is evident that at the present time physical amenities such as having a translator present, as well the training of the healthcare professionals to be able to make the most of the situation and see the patients in a holistic and biopsychosocial approach, reveal an important area to focus more on in practice as well as in research. Thus an increased availability of resources and training needs to be made available for both patients and healthcare professionals in order to ensure competence in accessing healthcare services.
\end{abstract}

Keywords: Cultural Competency; Health Services Accessibility; Health Services Needs and Demand; Humans; Language (Source: MeSH, NLM).

\section{Introduction}

About the Author: Nicole Tan is currently a thirdyear medical student at the Guy's, King's and St Thomas's Medical school, King's College London. She took a year out of the 5-year MBBS programme last year to intercalate in Clinical Pharmacology and Translational Medicine. society.
The world is becoming progressively more diverse, with variances in age, socioeconomic status, religion, personality, as well as in race and ethnicity. This change in ethnicity and identity over time affects the very constructs of society, causing us to question how well we are embracing other cultures, without losing the diversity and traditions each represent, but also how much change should we make if we are to make an equal

One of the many ways in which culture can be defined is as a group of individuals who have similar attitudes, beliefs and values, both in the group and when on their own.' Hence this suggests that culture forms from a sharing of commonality in the way you behave and perceive, rather than specifically your ethnic or racial heritage. Beagan ${ }^{2}$ suggests that there is a power and dominance which features in today's society as everyday racism, a term first used by Philomena Essed, thus perpetuating the inequality and normalization of asserting authority over others deemed different. This highlights the need for guidance and research into how to appreciate the multicultural world we live in today and augment equality in all aspects of society, as we still do live in an unequal and discriminative society.

Nevertheless, cultural diversity is present, and is an ever debateable issue within healthcare. With both the population of patients and healthcare professionals becoming more diverse, the National Health Service (NHS) is becoming more and more challenged to ensure good access and provision of excellent medical care. The NHS constitution sets out what the NHS as a service should provide for the patient i.e. access to free healthcare to everyone who clinically needs it, and the rights for the staff who provide this care. This in theory helps to tackle any issues about trying to increase public knowledge about what they can access, and what to expect from the NHS. However, as discussed in a report in the Health Care Analysis, ${ }^{3}$ there are still many aspects which need to be addressed with regards to Human Rights alongside the NHS, including the right to refuse treatment on religious grounds, and the right as a healthcare professional to opt out of some treatment procedures. It is evident that though there is research, there is insufficient delivering of excellence in cultural competence and society is already witnessing effects. ${ }^{4}$

Therefore, the aim of this article is to highlight the different aspects of current issues surrounding multiculturalism in healthcare, particularly to appreciate what the world of medicine has to face when attempting to tackle multiculturalism in healthcare and also to reveal what provisions are already in use and how well they work, from both the view of the patient and healthcare professional.

Search Strategy, Selection Criteria and Qualitative Analysis

This literature review was conducted using online databases to cover all health science articles, of which two of them (Medline and PubMed) are based around biomedical literature, whilst 
the other two (Scopus and Web of Science) look more broadly at health science. The articles were chosen using a search with the following terms: (1) cultural diversity of the patient, (2) multicultural attitude to health, (3) cross cultural communication barriers, (4) cultural diversity of the healthcare professionals/physicians, and (5) cultural competence training.

Other criteria included being written in English and also being within the last ten years, from 2005 until 2015, in order to ensure that the most up to date research and relevant, were included. As this revealed thousands of articles, the selection involved reading the title and the abstract of the articles, then choosing the first three results for each search, on the basis that these are immediately relevant to the themes of diversity of the patients and healthcare professionals and healthcare provisions.

Once selected, the articles were run through NVivo 10 (Victoria, Australia) ${ }^{5}$ to enable qualitative analysis based on their original search criteria. The qualitative analysis involved a content analysis, which was performed on each article for each search term using a summative approach, thus the article was read and if any quote was inferred to be related to a theme, then the article was included in that theme's results and discussion. If an article was found via the first search term but also contains information about a second search term, then the article could be used for both themes.

\section{Search Results}

Over the period of January and February 2015, a total of 60 articles were selected and analysed. The number sixty was chosen to enable the top three articles in each search of each key term in each database, to be considered in the analysis. Table 1 shows all of the articles selected as well as which search terms can be found within each article and hence how they have been used in the discussion below.

of the 60 articles, there are 32 articles which are relevant to the theme of cultural diversity of the patient, 25 articles for the topic of multicultural attitudes to health, 20 articles appropriate to the query cross cultural communication barriers, 25 articles for cultural diversity of the healthcare professional, and 33 articles about cultural competence training.

\section{Cultural Diversity of Patients}

There are many characteristics surrounding the social and cultural diversity of a patient, and so it has become an important aspect of medical communication, forming a basis for decisions, expectations and acceptability in both eyes of the healthcare professional and the patient. ${ }^{6}$

In today's society, there is an increasing proportion of people who have moved from their country of origin to be where they are today, and in a few cases immigrants can be found to have health problems. This can be something that they have carried with them from their country of origin, or as a result of the process of migration and the stress of the new environment, leading to biopsychosocial health problems, ${ }^{7}$ including post-traumatic stress disorder, depression, ${ }^{8}$ asthma, ${ }^{9}$ communicable diseases, and reproductive complications. ${ }^{10}$ Migrants may have to undergo rigorous checks before being able to migrate into e.g. Australia, or may be part of the new rich population where immigration is planned, thus a phenomena known as the healthy migrant effect can also be seen where they enjoy better health. However, as the migrants acculturate into society, the lack of access to resources, in particular healthcare, may lead to a decline in their health and thus a higher incidence of disease. ${ }^{11,12}$ There are two main groups of factors that are thought to lead to the health disparities that can be seen: differences between the country of origin and the country migrated to with regards to language, culture and environment; and post arrival socioeconomic factors, including marginalization, lack of access to programs and poverty. ${ }^{13}$

Patient diversity is reflected in physical aspects as well as sociocultural aspects. Haskell and Segal highlighted the anatomical variability, ${ }^{14}$ whilst Minkov and Bond looked at the genetic polymorphisms in the way that some nations have higher proportions of people who carry a polymorphism associated with risk taking and impulsivity. ${ }^{15}$

Cultural diversity also exists in beliefs, religion and spirituality, which can be found to play a role in the way that healthcare is perceived. One of the examples of religious practices which conflicts with medical care, but is a common practice amongst cultures in the Middle East and Africa, is female genital mutilation (FGM). This is seen as a rite of passage for many young girls, to enable her to become a woman, even though it is illegal in many countries, and there are many physical and psychological consequences for the girl who has this done to. Yet it is estimated that 130 million girls and women have undergone FGM and many more are at risk having a sort of the procedure undertaken. ${ }^{16}$

Religion and spiritual beliefs have been seen to affect the health of the patient in a different manner at the same time, with more involvement being associated with lower blood pressure, fewer strokes, lower rates of heart disease, ${ }^{17}$ a reduced access to hospice care and less aggressive medical interventions in end of life care. ${ }^{18}$ Palliative care patients who were well supported religiously focused on positive outcomes, were more hopeful despite their despair, and had a higher coping capacity. ${ }^{18}$ There are also different views of acceptable treatment that can be given. Tomlinson and Stott ${ }^{19}$ investigated international attitudes of assisted dying in dementia, revealing differences in views with regards to gender, ethnicity, education, religion and geographical location, whilst another study ${ }^{20}$ reported that though a need may be recognised, e.g. with kidney transplantation, such ideas are simply not considered.

Some more of the aspects that may be encountered with regards to culture include fasting and taking medicines, and also the origins of the ingredients in the medicine itself, which would impact the compliance to the medication prescribed. Help is also often sought from traditional and alternative medicines as well as from faith healers. This needs to be discussed with the patient, providing more information, and finding alternatives to the drug formulation to ensure that the patient is viewed as a person, in a pluralistic society. ${ }^{17,21}$

Patterns of discrimination were found in many aspects of socially influenced measures of wellbeing including housing and 
Table 1. Studies Used in the Content Analysis and Pertaining Themes

\begin{tabular}{|c|c|c|c|c|c|}
\hline Study & $\begin{array}{l}\text { Cultural diversity } \\
\text { of patients }\end{array}$ & $\begin{array}{l}\text { Multicultural attitude } \\
\text { to health }\end{array}$ & $\begin{array}{c}\text { Cross cultural } \\
\text { communication barriers }\end{array}$ & $\begin{array}{l}\text { Cultural diversity of } \\
\text { healthcare professionals }\end{array}$ & $\begin{array}{c}\text { Cultural competence } \\
\text { training }\end{array}$ \\
\hline Abbott et al (2014) & & & $\checkmark$ Web of Science & & $\checkmark$ \\
\hline Abe et al (2014) & & $\checkmark$ & & $\checkmark$ Web of Science & \\
\hline Aggarwal et al (2013) & $\checkmark$ Scopus & & & $\checkmark$ & $\checkmark$ \\
\hline Ahmad et al (2014) & & $\checkmark$ PubMed & & & $\checkmark$ \\
\hline Amati et al (2015) & & $\checkmark$ & & $\checkmark$ Scopus & \\
\hline Ballantyne et al (2013) & & $\checkmark$ & $\checkmark$ Medline & & \\
\hline Chien et al (2013) & $\checkmark$ & $\checkmark$ & $\checkmark$ Medline & & \\
\hline Cunningham et al (2014) & $\checkmark$ Web of Science & & $\checkmark$ & $\checkmark$ & $\checkmark$ \\
\hline Daher et al (2015) & $\checkmark$ Web of Science & $\checkmark$ & & $\checkmark$ & $\checkmark$ \\
\hline Dahl-Michelson (2015) & & & & $\checkmark$ & $\checkmark$ Web of Science \\
\hline Davison et al (2014) & $\checkmark$ Web of Science & $\checkmark$ & & & \\
\hline Dawson et al (2015) & $\checkmark$ & & & $\checkmark$ & $\checkmark$ Web of Science \\
\hline DelVecchio et al (2014) & $\checkmark$ PubMed & & & & $\checkmark$ \\
\hline Dewell et al (2013) & $\checkmark$ & $\checkmark$ Scopus & & & $\checkmark$ \\
\hline Fisher et al (2013) & $\checkmark$ & $\checkmark$ Medline & & & $\checkmark$ \\
\hline French et al (2014) & & & & $\checkmark$ PubMed & \\
\hline Giacco et al (2014) & $\checkmark$ & & $\checkmark$ Web of Science & $\checkmark$ & \\
\hline Hart et al (2013) & $\checkmark$ & & $\checkmark$ & $\checkmark$ Web of Science & \\
\hline Haskell et al (2014) & $\checkmark$ & $\checkmark$ Medline & & $\checkmark$ & $\checkmark$ \\
\hline Higginbottom et al (2014) & $\checkmark$ & $\checkmark$ Web of Science & & & \\
\hline Holland (2015) & $\checkmark$ & & & $\checkmark$ & $\checkmark$ Scopus \\
\hline Hooker et al (2011) & $\checkmark$ Medline & & & & $\checkmark$ \\
\hline Iglehart (2014) & & & & $\checkmark$ Medline & \\
\hline Jacob (2014) & $\checkmark$ PubMed & & & $\checkmark$ & $\checkmark$ \\
\hline Kantaris et al (2014) & $\checkmark$ & & $\checkmark$ PubMed & & \\
\hline Klein et al (2009) & $\checkmark$ Medline & & & & $\checkmark$ \\
\hline Koffman (2014) & $\checkmark$ & $\checkmark$ & $\checkmark$ & $\checkmark$ Web of Science & \\
\hline Komaric et al (2012) & $\checkmark$ Medline & & & & $\checkmark$ \\
\hline Kulwicki et al (2015) & $\checkmark$ & $\checkmark$ Scopus & $\checkmark$ & & \\
\hline Leyerzapf et al (2014) & & & & $\checkmark$ PubMed & \\
\hline Liu et al (2015) & & & $\checkmark$ scopus & & $\checkmark$ \\
\hline Logan et al (2015) & & & $\checkmark$ Scopus & & \\
\hline Long (2014) & & & & & $\checkmark$ Medline \\
\hline López-Sierra et al (2015) & $\checkmark$ PubMed & & & & $\checkmark$ \\
\hline Merchant et al (2010) & & $\checkmark$ & & $\checkmark$ Medline & $\checkmark$ \\
\hline Minkov et al (2015) & $\checkmark$ Scopus & & & & \\
\hline Mobula et al (2015) & & & & $\checkmark$ & $\checkmark$ Web of Science \\
\hline Nicholas et al (2014) & & $\checkmark$ & $\checkmark$ Medline & & $\checkmark$ \\
\hline Nichols et al (2015) & & & & $\checkmark$ & $\checkmark$ scopus \\
\hline Nunez-Smith et al (2009) & & & & $\checkmark$ Medline & \\
\hline Ochoa et al (2014) & $\checkmark$ & $\checkmark$ PubMed & $\checkmark$ & & \\
\hline Palmer et al (2014) & $\checkmark$ & & & & $\checkmark$ Medline \\
\hline Patel et al (2014) & $\checkmark$ & & & & $\checkmark$ Medline \\
\hline Perry et al (2015) & $\checkmark$ & & & & $\checkmark$ PubMed \\
\hline
\end{tabular}


Table 1. (Continued)

\begin{tabular}{|c|c|c|c|c|c|}
\hline Study & $\begin{array}{l}\text { Cultural diversity } \\
\text { of patients }\end{array}$ & $\begin{array}{l}\text { Multicultural attitude } \\
\text { to health }\end{array}$ & $\begin{array}{c}\text { Cross cultural } \\
\text { communication barriers }\end{array}$ & $\begin{array}{l}\text { Cultural diversity of } \\
\text { healthcare professionals }\end{array}$ & $\begin{array}{c}\text { Cultural competence } \\
\text { training }\end{array}$ \\
\hline Popper-Giveon et al (2014) & $\checkmark$ & & $\checkmark$ & $\checkmark$ PubMed & \\
\hline Read et al (2014) & & $\checkmark$ Web of Science & & & \\
\hline Rogers et al (2014) & $\checkmark$ & $\checkmark$ Scopus & $\checkmark$ & & $\checkmark$ \\
\hline Saloner et al (2014) & & $\checkmark$ PubMed & & & \\
\hline Saunders et al (2015) & & & & & $\checkmark$ scopus \\
\hline Shannon (2014) & $\checkmark$ & $\checkmark$ & $\checkmark$ Web of Science & & \\
\hline Shefer et al (2012) & & $\checkmark$ Medline & $\checkmark$ & & \\
\hline Simpkins et al (2014) & & & & & $\checkmark$ PubMed \\
\hline Simplican et al (2014) & & & $\checkmark$ scopus & & \\
\hline Smith et al (2014) & $\checkmark$ & $\checkmark$ Web of Science & & & $\checkmark$ \\
\hline Strong et al (2015) & & $\checkmark$ & & $\checkmark$ & $\checkmark$ PubMed \\
\hline Stütz et al (2015) & & & & $\checkmark$ Scopus & $\checkmark$ \\
\hline Tomlinson et al (2014) & $\checkmark$ scopus & $\checkmark$ & & $\checkmark$ & \\
\hline Vaughan et al (2015) & & & & $\checkmark$ Scopus & \\
\hline Vidaeff et al (2015) & & $\checkmark$ & $\checkmark$ PubMed & & $\checkmark$ \\
\hline Williams et al (2015) & & & $\checkmark$ PubMed & & \\
\hline
\end{tabular}

healthcare, with persistent inequalities and disparities across medical specialties..$^{22}$ Hooker and Noonan ${ }^{23}$ exposed the hegemonistic western culture, in which medical humanities marginalise diversity and different cultural traditions, making them a negative deviation from the norm of Anglo-American and European culture, and stigmatising this as different and therefore devalued, thus perpetuating the disadvantage of not being from the dominant culture. Cunningham et $\mathrm{al}^{24}$ emphasises this point with research into prior studies finding that Blacks perceive discrimination when Whites do not, and attribute the cause differently, thus highlighting the importance of perception from both patient and healthcare professional.

Dynamic sizing for each individual patient has been found to be a key part of the balancing act, between not defining difference using universal commonalities, and highlighting the differences which leads to discrimination. This can be seen to be complicated by multiple ethnic groups, ambiguity of the culture, simultaneity of multiple ethnic groups, fluidity, and misapplication of an individual's cultural orientation relative to the cultural groups. ${ }^{22}$ Thus cultural differences between the patient and physician can be problematic, and this leads to a decreased quality of care and a higher burden of disease in these individuals."

\section{Multicultural Attitude to Health}

The priority which people place on healthcare and medicine varies with ethnicity, religion, socioeconomic status and access. This includes whether the patient is or isn't involved in the decisions about their healthcare. The idea of "shared decision making" where patients are involved in how to manage the situation uses the basis of ethical principle of patient autonomy and patient centred care seen in Western medicine, although Vidaeff, Kerrigan and Monga ${ }^{25}$ reported that some cultures including Middle East or Eastern Europeans, do not inform the patient of terminal diagnoses as this may accelerate death or "cruelly burden the time left to live", hence do not value patient autonomy over culture and beneficence.

The cause of symptoms presented are also debated from the various models, it can be described as supernatural ${ }^{8,26}$ and therefore requiring religious aid such as prayers and complementary treatments, or biomedical that may require medication or surgery. Read et $\mathrm{a}^{27}$ researched into the use of complementary and alternative medicine by patients of various ethnicities in Canada, finding that many women endorsed the complementary and alternative medicine for infertility, focusing mainly on acupuncture and Chinese or other herbal medicines, as well as medicines that derived from their cultural backgrounds. This pursuit of their own culture's traditional healing methods may not be seen as acceptable or understandable by healthcare professionals in the dominant culture, ${ }^{27}$ revealing the strong paternalistic and biomedical feature of some clinicians which may discourage patients from seeking healthcare. ${ }^{28}$ Thus the act of introspection and deconstruction of a situation, by looking at the way in which human biology may be different e.g. body shape and build, how ethnicity is perceived by the individual, and the associated societal preconceptions; so as not to be inadvertently prejudiced, is key in order to make available a patient-centred model of biopsychosocial care..$^{14,29}$

Shefer et $\mathrm{a}^{26}$ explored the views and attitudes of healthcare: self-critical (biomedical), medical critical, traditional and integrating; highlighting the different aspects to consider when trying to engage people and the relative importance of healthcare to each individual. These points of view can vary across a variety of topics: medications seen as both harmful and helpful, ${ }^{30}$ assisted dying and euthanasia, ${ }^{19,31,32}$ and what counts as an illness within the society. ${ }^{26}$ This may lead to the development of a lack of understanding of their own health, 
for example with regards to diabetes terminology, 33 fear and misunderstandings with organ donation and transplantation ${ }^{20}$ and ultimately the incompletion of treatment. ${ }^{34}$

Discrimination from their community is another aspect which can influence an individual from seeking treatment. Stigma about mental disorders, ${ }^{8,26,35}$ sexual and reproductive health, ${ }^{36}$ and HIV $^{37}$ may set in motion a range of cultural responses; some may feel the need to hide the problem due to fear of rejection by their family or local community, or may fear the impact on the family's reputation and further prospects by the family thereafter. ${ }^{20,36}$ The imbalance of gender can also dictate whether treatment is sought, with males holding most of the power. ${ }^{36,38,39}$ on the other hand, the community can provide much of the needed support and advice, whether the relatives are living nearby or via the telephone or the internet, enabling them to gain experiential knowledge as well as learn where to purchase products from their own culture, for example foods from a specialty ethnic supermarket which have been religiously or culturally sanctioned at the time of pregnancy. ${ }^{12}$

Patients report many different experiences from the healthcare system, with some reporting that there was discrimination, and a lack of understanding about their culture including health related beliefs and dietary requirements ${ }^{40}$ as well as facing many access issues, a lack of support and education about available services. ${ }^{10,36}$ Another issue that was highlighted was about trust and confidentiality; many do not trust the healthcare services for fear of being reported or stigmatised. ${ }^{8,20,26,36}$ Others reported that they were felt to be respected, and that their care was tailored to their needs. ${ }^{40,47}$

\section{Cross Cultural Communication Barriers}

There have been many reports of barriers to accessing healthcare, including language barriers and general poor communication between the healthcare professional and the patient and their family. This has been seen to lead to a number of incidents where the patient and the healthcare professional are unsatisfied with the care given and received. $7,8,25,35,36,38,39,42,43$ The impact of different languages and translation in this poor communication reduces the chance of receiving good treatment and adherence to the treatment set, but interpreting services, via telephone or face-to-face are yet another demanded resource that the healthcare system is unable to provide to the necessary standards. ${ }^{8,30,35,39,43}$ Many research papers also review the perceptions of the healthcare system from a variety of cultural backgrounds. Here they look predominantly at the differences between their home country's healthcare system and the new healthcare system of the country they have moved to, showing that there are changes in the way the healthcare professionals behave and act around the patient, ${ }^{44}$ especially the spoken and body language aspects of communication. ${ }^{25,32}$

There are also circumstantial factors which play a part in acting as barriers such as financial status and socioeconomic status that have been found to result in low service use. ${ }^{7,8,36,38}$ In addition, experiential factors such as long waiting times, care that does not meet their standards, and a lack of transport to access the healthcare can reduce service use. ${ }^{7,35,36,38} \mathrm{~A}$ lack of knowledge about what the patient can expect to receive or even how to begin to access healthcare has also been shown to act as a barrier. ${ }^{7,45}$ Such factors affect social inclusion which has been found to promote wellbeing, mental health, and decision making capacity and discourages discrimination and stigma, ${ }^{46}$ and also increases the anxiety of the situation, for fear of being stigmatised or outcast from society for attempting to access healthcare, resulting in less willingness to access the care they need. ${ }^{26,38,39,47,48}$

One of the other barriers to healthcare is how healthcare professionals are facing multiculturalism in healthcare which has resulted in "culturally competent care [that was] misguided or insufficient". ${ }^{44}$ There have also been notable shortcomings in policy and regulation in trying to achieve cultural competen$\mathrm{ce}^{42}$ on top of issues such as stereotyping, ${ }^{25}$ time pressures in accessing the information that the healthcare professionals require ${ }^{45}$ and lack of training. ${ }^{43}$

\section{Cultural Diversity of Healthcare Professionals} The healthcare professional workforce can also be seen to be diverse, with the number of physicians from migrant backgrounds increasing. Giacco, Matanov and Priebe $^{8}$ found that of the mental health services sampled, seventy six per cent had members of staff with migrant backgrounds, and this was frequently considered to be an effective way to address the migrant population of patients. ${ }^{8,49}$ Many studies reveal that though this may be happening, the physician population is still majorly under representative of the patient population with regards to ethnicity although gender is imbalanced to0.43,49-51 The make-up of the population of medical specialties varies, with physiotherapists being divided by gender and so the male domination of sports and manual therapy, ${ }^{52}$ and that there is a statistically significant difference between the characteristics of a physician in the surgical speciality in comparison to one in a non-surgical specialty, with regards to gender and political identity. ${ }^{53}$ Physician attitude is also different with regards to their views on assisted dying and dementia, ${ }^{19}$ truthfulness to the patient and their family ${ }^{31}$ and their perceptions of end of life care. ${ }^{37}$

The performance of medical students has been shown to be dependent on their ethnicity and the culture that they are in, with research revealing that in the UK, those who are non-White are more likely to have a lower achievement, although networking factors had a greater impact on grades than ethnicity. ${ }^{54}$

Healthcare professionals with a migrant background disclose diverse experiences in the workplace. Some report positive or supportive relationships, ${ }^{37,55}$ whilst others report negative, discriminatory and upsetting relationships with their colleagues. ${ }^{55,56}$ Leyerzapf et al57 found that a cultural minority ethnicity of a physician is seen by specialists and heads of departments to possibly cause a delay in the career paths of the students, although they are also seen to participate less in extracurricular activities including doing internships and PhD research which may also impact on their career. It was also noted that "cultural minority respondents stressed that their experience of constantly standing out in a negative way because of their cultural background" making what they felt as valuable, as something to be ashamed of. This lack of understanding of different cultures is being seen to impact not only the patient-physician relationship, but also the relationship 
between healthcare professionals ${ }^{55}$ when their cross-cultural experiences could be used as an educational benefit, for other healthcare professionals to learn from. ${ }^{32,58}$

\section{Cultural Competence Training}

There has been a lot of research into the development of resources to be used to help with cultural competence. This includes the use of medical interpreters available in person, the ideal scenario, or via telephone or videoconference ${ }^{59}$ as well as providing resources in a range of languages and a variety of modes of communication, e.g. emails, leaflets, phone calls..$^{44,60}$

There are many training models, teaching how to make effective communication for a range of audiences, to enable the healthcare professional to reflect on how to give the patient the information that they need and assess patient satisfaction. ${ }^{28,42,44}$ Training should take place using a variety of teaching methods, for example with acting, esimulationg, ${ }^{61}$ or cultural immersion experiences and not just via lectures and readings. ${ }^{62}$ With acting, by performing the other role, this enlightens the individual by stepping into the other's shoes, and giving them experience that they can draw on when in a consultation. ${ }^{33,42,52}$ The Interprofessional Community Health and Education Exchange (iCHEE) sessions are another good example of training in a multidisciplinary team, where four five-hour-long sessions in the community are used to discuss how they would tackle cultural and medical issues in physically inconvenient and resource poor environments. ${ }^{13}$ As Saunders, Haskins and Vasquez ${ }^{63}$ highlight the elusiveness of cultural competence, they indicate the problems with the training provided including instructors feeling uncomfortable in exploring cultural competence and the issue of ascertaining where to draw the line as to when to say that someone is culturally competent, hence revealing a lifetime "journey to an elusive goal". A lack of knowledge of what exactly to teach was also stressed as an issue. ${ }^{64}$ Another issue is the complexity of the situation, for example with female genital mutilation (FGM) where the healthcare professionals need to be taught with how to be sensitive and non-discriminatory but at the same time, know to report the incidence of FGM and the complications and risks involved with it. ${ }^{16}$

One of the areas of concern involves the recognition of cultural conceptual tensions and the difficulty in adapting the situation without being seen as discriminative. ${ }^{63}$ This promotion of stereotyping in order to teach about ethno-specific groups, can be seen to go against the purpose of the training. In addition to this, the very way in which we assess health, particularly mental health, is Westernised, and what can be seen to be normal in one culture, may be seen as abnormal in another, hence revealing the need for better tools for assessment. ${ }^{6}$

National initiatives should also be used to the full extent, with the enrolling of more ethnic minority medical students ${ }^{50}$ and the adaptations to the medical school curriculum to reflect the changing society ${ }^{58}$ and invest in more culturally competent care. ${ }^{24}$ Giacco, Matanov and Priebe ${ }^{8}$ revealed the importance of organisational flexibility, interpreting services, culturally aware staff, psychoeducational programs, ensuring stable relationships with patients and having clear guidelines of what people are entitled to, in order to have good practice within healthcare.
To improve access to the healthcare system, there need to be bridges, whether this is by the community mental health team ${ }^{65}$ or the learning of another language to increase the sense of value. ${ }^{55}$ Alternatively, proactively recognising their healthcare by including non-biomedical explanation models of illness or revealing implicated ingredients in a medicine etc., helps to ensure patient autonomy and encourages the access of healthcare by improving the experience of seeming helpful as opposed to distrustful and manipulative. ${ }^{17,21}$

\section{Conclusion}

In conclusion, this literature review of sixty articles has highlighted the importance of the variety in culture and being able to find the right way to deal with the issues surrounding intercultural communities. With a multitude of ways in which the patient and the healthcare professional can differ, whether it is the physical health status, psychological beliefs, or schemas teamed with experience; this all leads to conflicting views on aspects within healthcare. One of the challenges with tackling intercultural competence by the healthcare professional is attempting to find the balance between cultural distinctiveness and discrimination; dynamic sizing for each individual patient is suggested, a process riddled with many complications and can ultimately be detrimental to the health of the patients themselves. As seen in this article, which only samples a handful of the research, there is a lot of research that has already been undertaken in this field, with a recognisable need for this to be translated into practice and into the healthcare systems. Resources need to be made readily available for everyone to use, and the adaptation of the healthcare system to an ever-changing society needs to be commenced. This includes having the idyllic interpreter who is capable of explaining medical terminology, being present at all times when the patient accesses healthcare to enable continuity of care. Healthcare professionals need to be able to bridge the gap between the healthcare systems and the community. Healthcare professionals themselves need to learn to recognise the differences in themselves, between fellow healthcare professionals and the patient population, and reflect this back into their work, creating time for those that need it the most, being more open to diverse methods of seeing and treating patients, making the most of their heritage and ensuring good medical practice. 


\section{References}

1. Kai J, editor. Ethnicity, health and primary care. Oxford: Oxford University Press; 2003.

2. Beagan BL. 'Is this worth getting into a big fuss over?' Everyday racism in medical school. Med Educ. 2013 Oct;37(10):852-60.

3. McHale JV. Faith, belief, fundamental rights and delivering health care in a modern NHS: an unrealistic aspiration? Health Care Anal. 2013 Sep;21(3):22436.

4. Jacobs E, Chen AH, Karliner LS, Agger-Gupta N, Mutha S. The need for more research on language barriers in health care: a proposed research agenda. Millbank Q. 2006;84(1):111-33.

5. SR International Pty Ltd. NVivo qualitative data analysis Software. Version 10; 2012.

6. Aggarwal NK, Desilva R, Nicasio AV, Boiler M, Lewis-Fernández R. Does the Cultural Formulation Interview for the fifth revision of the diagnostic and statistical manual of mental disorders (DSM-5) affect medical communication? A qualitative exploratory study from the New York site. Ethn Health. 2015;20(1):1-28.

7. Kantaris $\mathrm{M}$, Theodorou M, Galanis $\mathrm{P}$, Kaitelidou D. Access and utilisation of health services by domestic helpers in Cyprus. Int J Health Plann Manage. 2014 Oct-Dec;29(4):e383-93.

8. Giacco D, Matanov A, Priebe S. Providing mental healthcare to immigrants: current challenges and new strategies. Curr Opin Psychiatry. 2014 Jul;27(4):282-8.

9. Patel MR, Thomas LJ, Hafeez K, Shankin M, Wilkin M, Brown RW. Study protocol for improving asthma outcomes through cross-cultural communication training for physicians: a randomized trial of physician training. BMC Med Educ. 2014 Jun 16;14:118.

10. Ochoa SC, Sampalis J. Risk perception and vulnerability to STIs and HIV/ AIDS among immigrant Latin-American women in Canada. Cult Health Sex. 2014;16(4):412-25.

11. Komaric N, Bedford S, van Driel ML. Two sides of the coin: patient and provider perceptions of healthcare delivery to patients from culturally and linguistically diverse backgrounds. BMC Health Serv Res. 2012 Sep 18;12:322. 12. Higginbottom GM, Vallianatos H, Forgeron J, Gibbons D, Mamede F, Barolia $R$. Food choices and practices during pregnancy of immigrant women with high-risk pregnancies in Canada: a pilot study. BMC Pregnancy Childbirth. 2014 Dec 3;14:370.

13. Palmer VS, Mazumder R, Spencer PS. Interprofessional global health education in a cosmopolitan community of North America: the iCHEE experience. Acad Med. 2014 Aug;89(8):1149-52.

14. Haskell BS, Segal ES. Ethnic and ethical challenges in treatment planning: dealing with diversity in the 21st century. Angle Orthod. 2014 Mar;84(2):380-2. 15. Minkov M, Bond MH. Genetic polymorphisms predict national differences in life history strategy and time orientation. Pers Individ Dif. 2015 Apr;76:20415 .

16. Dawson A, Turkmani S, Fray S, Nanayakkara S, Varol N, Homer C. Evidence to inform education, training and supportive work environments for midwives involved in the care of women with female genital mutilation: a review of global experience. Midwifery. 2015 Jan;31(1):229-38.

17. Daher M, Chaar B, Saini B. Impact of patients' religious and spiritual beliefs in pharmacy: from the perspective of the pharmacist. Res Social Adm Pharm. 2015 Jan-Feb;11(1):e31-41.

18. López-Sierra HE, Rodríguez-Sanchez J. The supportive roles of religion and spirituality in end-of-life and palliative care of patients with cancer in a culturally diverse context: a literature review. Curr Opin Support Palliat Care. 2015 Mar;9(1):87-95.

19. Tomlinson E, Stott J. Assisted dying in dementia: a systematic review of the international literature on the attitudes of health professionals, patients, carers and the public, and the factors associated with these. Int I Geriatrc Psychiatry. 2015 Jan;30(1):10-20.
20. Davison SN, Jhangri GS. Knowledge and attitudes of Canadian First Nations people toward organ donation and transplantation: a quantitative and qualitative analysis. Am J Kidney Dis. 2014 Nov;64(5)781-9.

21. Jacob KS. Insight in psychosis: an independent predictor of outcome or an explanatory model of illness? Asian J Psychiatr. 2014 0ct;11:65-71.

22. Good MJ, Hannah SD. "Shattering culture": perspectives on cultural competence and evidence-based practice in mental health services. Transcult Psychiatry. 2015 Apr;52(2):198-221.

23. Hooker C, Noonan E. Medical humanities as expressive of Western culture. Med Humanit. 2011 Dec 1;37(2):79-84.

24. Cunningham BA, Marsteller JA, Romano MJ, Carson KA, Noronha GJ, McGuire MJ, et al. Perceptions of health system orientation: quality, patient centeredness, and cultural competency. Med Care Res Rev. 2014 Dec;71(6):559-79. 25. Vidaeff AC, Kerrigan AJ, Monga M. Cross-cultural barriers to health care. Southern Med J. 2015 Jan;108(1):1-4.

26. Shefer G, Rose D, Nellums L, Thornicroft G, Henderson C, Evans-Lacko S. 'Our community is the worst': the influence of cultural beliefs on stigma, relationships with family and help-seeking in three ethnic communities in London. Int J Soc Psychiatry. 2013 Sep;59(6):535-44.

27. Read SC, Carrier M, Whitely R, Gold I, Tulandi T, Zelkowitz P. Complementary and alternative medicine use in infertility: cultural and religious influences in a multicultural Canadian setting. J Altern Complement Med. 2014 Sep;20(9):686-92.

28. Strong KL, Folse VN. Assessing undergraduate nursing students' knowledge, attitudes, and cultural competence in caring for lesbian, gay, bisexual, and transgender patients. J Nurs Educ. 2015 Jan;54(1):45-9.

29. Smith MD, Jennings L, Lakhan S. International education and service learning: approaches toward cultural competency and social justice. Couns Psychol. 2014 Nov 3;42(8):1188-214.

30. Ballantyne PJ, Yang M, Boon H. Interpretation in cross-language research: tongues-tied in the health care interview? J Cross Cultural Cerontol. 2013 Dec;28(4):391-405.

31. Amati R, Hannawa AF. Physician-perceived contradictions in end-of-life communication: toward a self-report measurement scale. Health Commun. 2015;30(3):241-50.

32. Koffman J Servicing multi-cultural needs at the end of life. J Ren Care. 2014 Sep;40 Suppl 1:6-15.

33. Ahmad B, Ramadas A, Kia Fatt Q, Md Zain AZ. A pilot study: the development of a culturally tailored Malaysian Diabetes Education Module (MY-DEMO) based on the Health Belief Model. BMC Endoc Disord. 2014 Apr 8;14:31.

34. Saloner B, Carson N, Lê Cook B. Explaining racial/ethnic differences in adolescent substance abuse treatment completion in the United States: a decomposition analysis. J Adolesc Health. 2014 Jun;54(6):646-53.

35. Shannon PJ. Refugees' advice to physicians: how to ask about mental health. Fam Pract. 2014 Aug;31(4):462-6.

36. Rogers C, Earnest J. Sexual and reproductive health communication among Sudanese and Eritrean women: an exploratory study from Brisbane, Australia. Cult Health Sex. 2015;17(2):223-36.

37. Abe M, Turale S, Klunklin A, Supamanee T. Community health nurses' HIV health promotion and education programmes: a qualitative study. Int Nurs Rev. 2014 Dec;61(4):515-24.

38. Chien SC, Yeh YP, Wu JY, Lin $\mathrm{CH}$, Chang PC, Fang $\mathrm{CH}$, et al. Factors associated with intensiveness of use of child preventative health services in Taiwan: a comparative study between cross-cultural immigrant families and native-born families. Ethn Health. 2013;18(1):1-17.

39. Kulwicki A, Ballout S, Kilgore C, Hammad A, Dervartanian H. Intimate partner violence, depression, and barriers to service utilization in Arab American women. J Transcult Nurs. 2015 Jan;26(1):24-30.

40. Fisher J, Hinchliff S. Immigrant women's perceptions of their maternity care: a review of the literature. Part 2. Pract Midwife. 2013 Feb;16(2):32-4. 41. Dewell JA, Owen J. Addressing mental health disparities with Asian Ame- 


\section{Review}

rican clients: examining the generalizability of the common factors model. J Couns Dev. 2013;93(1): 80-87.

42. Liu X, Rohrer W, Luo A, Fang Z, He T, Xie W. Doctor-patient communication skills training in mainland China: a systematic review of the literature. Patient Educ Couns. 2015 Jan;98(1):3-14.

43. Hart PL, Mareno N. Cultural challenges and barriers through the voices of nurses. J Clin Nurs. 2014 Aug;23(15-16):2223-32.

44. Nicholas DB, Hendson L, Reis MD. Connection vs disconnection: examining culturally competent care in the neonatal intensive care unit. Soc Work Health Care. 2014;53(2):135-55.

45. Williams B, Perillo S, Brown T. What are the factors of organisational culture in health care settings that act as barriers to the implementation of evidence-based practice? A scoping review. Nurse Educ Today. 2015 Feb;35(2):e34-41. 46. Simplican SC, Leader C, Kosciulek J, Leahy M. Defining social inclusion of people with intellectual and developmental disabilities: an ecological model of social networks and community participation. Res Dev Disabil. 2015 Mar;38:18-29.

47. Abbott P, Reath J, Gordon E, Dave D, Harnden C, Hu W, et al. Ceneral Practitioner Supervisor assessment and teaching of Registrars consulting with Aboriginal patients - is cultural competence adequately considered? BMC Med Educ. 2014 Aug; 14:167.

48. Logan S, Steel Z, Hunt C. Investigating the effect of anxiety, uncertainty and ethnocentrism on willingness to interact in an intercultural communication. J Cross-Cult Psychol. 2015;46(1):39-52.

49. Popper-Giveon A, Liberman I, Keshet Y. Workforce ethnic diversity and culturally competent health care: the case of Arab physicians in Israel. Ethn Health. 2014;19(6):645-58.

50. Merchant J, Omary MB. Clogged up. Efforts to train more minority doctors remain stalled as population diversifies. Mod Healthc. 2010 Feb 22;40(8):24.

51. Iglehart JK. Diversity dynamics-challenges to a representative u.s. medical workforce. N Engl J Med. 2014 Oct 16;371(16):1471-4.

52. Dahl-Michelson T. Curing and caring competences in the skills training of physiotherapy students. Physiother Theory Pract. 2015 Jan;31(1):8-16.

53. French JC, O'Rourke C, Walsh RM. A current assessment of diversity characteristics and perceptions of their importance in the surgical workforce. J
Gastrointest Surg. 2014 Nov;18(11):1936-43.

54. Vaughan S, Sanders T, Crossley N, O'Neill P, Wass V. Bridging the gap: the roles of social capital and ethnicity in medical student achievement. Med Educ. 2015 Jan;49(1):114-23.

55. Nichols $\mathrm{P}$, Horner B, Fyfe K. Understanding and improving communication processes in an increasingly multicultural aged care workforce. J Aging Stud. 2015 Jan;32:23-31.

56. Nunez-Smith M, Pilgrim N, Wynia M, Desai MM, Jones BA, Bright C, et al. Race/ethnicity and workplace discrimination: results of a national survey of physicians. J Gen Intern Med. 2009 Nov;24(11)1198-204.

57. Leyerzapf H, Abma TA, Steenwijk RR, Croiset G, Verdonk P. Standing out and moving up: performance appraisal of cultural minority physicians. Adv Health Sci Educ Theory Pract. 2015 Oct;20(4):995-1010.

58. Stütz A, Green W, McAllister L, Eley D. Preparing medical graduates for an interconnected world: current practices and future possibilities for internationalizing the medical curriculum in different contexts. J Stud Int Educ. 2015;19(1)28-45.

59. Klein C0, Kajbjer K. eHealth tools for patients and professionals in a multicultural world. Stud Health Technol Inform. 2009;150:297-301.

60. Simpkins SD, Riggs NR. Cultural competence in afterschool programs. New Dir Youth Dev. 2014 Dec;2014(144):105-17.

61. Perry A, Woodland L, Brunero S. eSimulation: a novel approach to enhancing cultural competence within a health care organisation. Nurse Educ Pract. 2015 May; $15(3): 218-24$.

62. Long T. Influence of international service-learning on nursing student self-efficacy toward cultural competence. J Nurs Educ. 2014 Aug;53(8):474-8. 63. Saunders JA, Haskins M, Vasquez M. Cultural competence: a journey to an elusive goal. J Soc Work Educ. 2015;51(1):19-34.

64. Holland AE. The lived experience of teaching about race in cultural nursing education. J Transcult Nurs. 2015 Jan;26(1):92-100.

65. Mobula LM, Okoye MT, Boulware LE, Carson KA, Marstellar JA, Cooper LA. Cultural competence and perceptions of community health workers' effectiveness for reducing health care disparities. J Prim Care Community Health. 2015 Jan; 6(1)10-5.

\section{Acknowledgments}

Student Selected Component Intercultural Competencies in Medicine, Guy's, King's and St Thomas' (GKT) School of Medicine at King's College London.

\section{Conflict of Interest Statement ct Funding}

The authors have no funding, financial relationships, or conflicts of interest to disclose.

\section{Author Contributions}

Data collection, Data analysis and interpretation, Writing: NT, SL.

\section{Cite as:}

Tan N, Li S. Multiculturalism in healthcare: a review of current research into diversity found in the healthcare professional population and the patient population. Int J Med Students. 2016 Sep-Dec;4(3):112-9. 